\title{
Pneumocistose diagnosticada durante a gestação: relato de caso
}

\author{
Pneumocystosis diagnosed during pregnancy: case report
}

\author{
Luilson Geraldo Coelho Júnior ${ }^{1}$, Daniel Lima Souza ${ }^{1}$, Gabrielly Borges Machado ${ }^{2}$, \\ Juliana Siqueira Silva ${ }^{3}$, Maria Luíza Gonçalves dos Reis Monteiro ${ }^{4}$
} Coelho Júnior LG, Souza DL, Machado GB, Silva JS, Monteiro MLGR. Pneumocistose diagnosticada durante a gestação: relato de
caso / Pneumocystosis diagnosed during pregnancy: case report. Rev Med (São Paulo). 2016 jul-set.;95(3):146-51.

RESUMO: A infecção pelo vírus da imunodeficiência humana (HIV) cursa com um amplo espectro de apresentações clínicas, desde a fase aguda até a fase avançada da doença. Em indivíduos não tratados o tempo médio entre o contágio com o vírus e o surgimento da doença ocorre em torno de dez anos. $\mathrm{O}$ aparecimento de infecções oportunistas e neoplasias é definidor da Síndrome da Imunodeficiência Adquirida, sendo que nessas situações a contagem de linfócitos TCD4+ está abaixo de 200 células/ $\mathrm{mm}^{3}$. A pneumocistose, uma pneumonia fúngica, é causada pelo fungo Pneumocystis jirovecii. É considerada doença definidora da síndrome da imunodeficiência adquirida (SIDA), possui alta morbimortalidade, curso clínico grave; sendo uma causa comum de doença pulmonar oportunista em imunodeprimidos. Há poucos relatos de pneumocistose em pacientes gestantes infectadas pelo vírus da imunodeficiência humana (HIV), sendo que nesta parcela de pacientes, a pneumocistose é considerada uma doença rara. Descrevemos um caso em que a paciente não realizou Pré-Natal durante a gestação, apresentando como primeira manifestação clínica do HIV/SIDA, a pneumocistose.

Descritores: Pneumocystis jirovecii; Síndrome da imunodeficiência adquirida; Pneumonia por Pneumocystis; Infecções oportunistas; Candidíase bucal; Cuidado pré-natal.

\begin{abstract}
Human Immunodeficiency Virus (HIV) infection has a broad clinical presentation, from acute to advanced stages of the disease. The average time between infection and emergence of disease in untreated individuals is about ten years. Opportunistic infections and malignancies are AIDS-defining diseases and, in these situations, TCD4+ lymphocyte count is lower than 200 cells $/ \mathrm{mm}^{3}$. Pneumocystosis, a fungal pneumonia, caused by Pneumocystis jirovecii, is considered an AIDS-defining disease with high morbidity, mortality and severe clinical course. It is the most common opportunistic infection affecting immunocompromised patients. There are few pneumocystosis reports in HIV pregnant patients as, in this group, pneumocystosis is considered rare. We report a case in which patient had no prenatal care and presented pneumocystosis as the first clinical manifestation of HIV/AIDS.
\end{abstract}

Keywords: Pneumocystis jirovecii; Acquired immunodeficiency syndrome; Pneumonia, Pneumocystis; Opportunistic infections; Candidiasis, oral; Prenatal care.

1. Acadêmico de Medicina, Faculdade Atenas, Paracatu-MG, Brasil. E-mails: luilson-junior@hotmail.com, daniel.limasouza@gmail.com

2. Médica Infectologista, Faculdade Atenas, Paracatu-MG, Brasil. E-mail: gabyborges100@hotmail.com.

3. Médica Ginecologista, Faculdade Atenas, Paracatu-MG, Brasil. E-mail: ju_ptu@yahoo.com.br.

4. Médica Patologista, Departamento de Patologia Geral, Universidade Federal do Triângulo Mineiro, Uberaba, MG, Brasil. E-mail: marialuizapatologia@gmail.com.

Endereço para correspondência: Luilson Geraldo Coelho Júnior. Rua Bernardo Caparucho, 30, apart. 04. Bela Vista, Paracatu, MG, Brasil. E-mail: luilson-junior@hotmail.com. 


\section{INTRODUÇÃO}

síndrome da imunodeficiência adquirida (SIDA) atualmente é o principal fator de risco para desenvolvimento de pneumocistose, uma pneumonia fúngica, causada por Pneumocystis jirovecii ${ }^{1}$. O quadro de SIDA ocorre quando a contagem de linfócitos TCD4+ está abaixo de $200 \mathrm{cel} / \mathrm{mm}^{3}$; o que indica imunodepressão grave, sendo fator de risco para contrair infecções fúngicas, bacterianas e por protozoários ${ }^{1,2}$.

O trato respiratório tem sido o local mais acometido pelo HIV, desde as primeiras descrições da doença. Baseando-se em dados de autópsia, o pulmão foi o órgão afetado com uma incidência que variou de $100 \%$ no período inicial da pandemia da doença para $70 \%$ na era da terapia antirretroviral. Aproximadamente $70 \%$ dos pacientes portadores do vírus HIV, durante a evolução da doença apresentam alguma complicação pulmonar, principalmente de etiologia infecciosa ${ }^{3}$.

As infecções do trato respiratório inferior são 25 vezes mais comuns em pacientes com HIV do que na população em geral, acometendo até 90 casos a cada 1.000 pessoas/ano. Infecções pulmonares, não somente as infecções oportunistas correlacionadas com a SIDA são uma das causas mais frequentes de internação hospitalar em pacientes infectados pelo HIV; além disso, estas infecções continuam sendo uma das principais causas de morbimortalidade nesse grupo de pacientes ${ }^{4}$.

A pneumonia bacteriana é atualmente a causa mais frequente de infecções pulmonares em pacientes infectados pelo HIV, seguido pela pneumocistose e tuberculose. A pneumonia bacteriana é o diagnóstico de admissão mais comum. A pneumonia bacteriana pode ocorrer ao longo de todo o curso da infecção pelo HIV; no entanto, a incidência a infecção aumenta à medida que o número de linfócitos TCD4+ diminui. Assim como na população em geral, o Streptococcus pneumoniae (pneumococo) é a causa mais comum de pneumonia bacteriana adquirida na comunidade em adultos infectados pelo HIV $^{5,6,7}$.

Pessoas portadoras do vírus HIV podem apresentar infecção pelo Mycobacterium tuberculosis; quando a contagem de linfócitos TCD4+ diminui, a incidência de tuberculose aumenta. Pelo menos um terço das pessoas infectadas pelo vírus HIV em todo o mundo está infectado pelo Mycobacterium tuberculosis; logo, o HIV constitui em termos globais, o maior fator de risco para desenvolvimento de tuberculose. Além disso, a tuberculose é a principal causa de morte para as pessoas que vivem com o HIV em países de baixa e média renda ${ }^{8,9}$.

Em indivíduos com SIDA as complicações pulmonares de natureza infecciosa são importantes causas de morbimortalidade. A pneumocistose, uma pneumonia de etiologia fúngica, é uma complicação pulmonar em pacientes com HIV/ SIDA; todavia, esta infecção não é exclusiva de indivíduos infectados pelo HIV e que apresentam contagem de linfócitos TCD4+ baixa, outros fatores como transplante de medula óssea, terapia imunossupressora tornam o indivíduo propenso à infecção pelo fungo ${ }^{10}$.

A transmissão do fungo ocorre por via inalatória, sendo que a grande maioria das pessoas não desenvolve a doença permanecendo assintomática por longos períodos. Já em pacientes com imunossupressão, principalmente nos casos de SIDA, a infecção por Pneumocystis jirovecii ocorre por reativação do foco infeccioso endógeno pulmonar ou reinfecção exógena ${ }^{2}$.

O fungo lesa o pneumócito tipo I, aumentando a permeabilidade alvéolo-capilar, gerando edema intersticial e exsudato eosinofílico, ocupando todo espaço intraalveolar, comprometendo a troca gasosa; tais eventos levam à hipoxemia, insuficiência respiratória lenta e progressiva, tosse seca ${ }^{10,11}$.

O diagnóstico de certeza depende da identificação do $P$. jirovecii em secreções ou em tecido pulmonar, por meio de técnicas de coloração como Giemsa, Azul de Toluidina e Grocott. Os achados clínicos laboratoriais e radiográficos quando correlacionados auxiliam no diagnóstico; caracterizando, portanto, o diagnóstico de presunção por $P$. jirovecii ${ }^{12}$.

O aumento de desidrogenase láctica sérica (DHL) é um achado laboratorial frequente, porém pouco específico; a radiografia de tórax evidencia infiltrado intersticial difuso, porém a radiografia de tórax pode ser normal em até um quarto dos casos de pneumocistose; nessa situação, a tomografia computadorizada de tórax pode revelar atenuação pulmonar em vidro fosco ${ }^{12,13}$.

O Sulfametoxazol com Trimetoprim (SMX + TMP) é o tratamento de primeira escolha recomendado para pacientes infectados pelo HIV com pneumocistose leve, moderada ou grave; a associação de corticosteróides ao tratamento de pneumocistose moderada e grave apresentou redução importante nos índices de mortalidade ${ }^{14}$.

Os avanços no tratamento da infecção pelo HIV, bem como a profilaxia contra infecções oportunistas aumentaram a sobrevida dos pacientes soropositovos, alterando a incidência de pneumocistose, reduzindo infecções primárias e reinfecções por Pneumocystis jirovecii ${ }^{15}$.

Há poucos casos relatados na literatura sobre pneumocistose na gestação, o diagnóstico da infecção oportunista durante o período gestacional é raro. Sendo assim, descrevemos um caso de pneumocistose diagnosticado e tratado durante o período gestacional, em que a paciente não realizou as consultas do pré-natal e foi diagnosticada com HIV durante a gestação. Desse modo, este relato reforça a importância de se diagnosticar e tratar precocemente a mãe, visando um desfecho clínico favorável a gestante e ao feto. 


\section{RELATO DE CASO}

Paciente do sexo feminino, 29 anos, natural e procedente de Paracatu-MG, casada, negra, do lar, católica.

Paciente primigesta, nulípara, idade gestacional 20 semanas, não iniciou o Pré-Natal, deu entrada no pronto socorro do Hospital Municipal de Paracatu apresentando quadro de dispneia progressiva, há mais de uma semana, associada à febre baixa, tosse seca e perda ponderal.

Em relação aos antecedentes mórbidos pessoais negou alergias, cirurgias, internações e transfusões sanguíneas prévias. Não tabagista e não etilista. Negou uso de drogas ilícitas. Desconhece doenças de base e da infância.

Ao exame físico, paciente em regular estado geral, acianótica, porém com esforço respiratório e estertores em base pulmonar esquerda, $\mathrm{FR}=32 \mathrm{irpm}, \mathrm{S} 02=95 \%$.
À oroscopia lábios secos, mucosa oral e jugal integra, hipocorada e desidratada; presença de hiperemia e placas esbranquiçadas em pilar amigdaliano e orofaringe.

Foi admitida na enfermaria de clinica médica, em leito de isolamento, devido o diagnóstico de HIV/AIDS além de quadro sugestivo de pneumocistose e candidíase oral.

Solicitado, tomografia computadorizada de tórax (TC de Tórax), contagem de linfócitos TCD4+, carga viral, desidrogenase lática (DHL) e pesquisa de bacilos álcool-ácido resistentes (BAAR) no escarro três amostras. Resultados: $\mathrm{DHL}=380,7 \mathrm{U} / \mathrm{L}, \mathrm{BAAR}$ negativo nas três amostras, TC de tórax evidenciou opacidade em vidro fosco em ambos os pulmões com predomínio em terço médio, achado compatível com pneumocistose (Figura 1), carga viral 993 cópias/mL, linfócitos TCD4+ 110 células/ $\mu 1$.

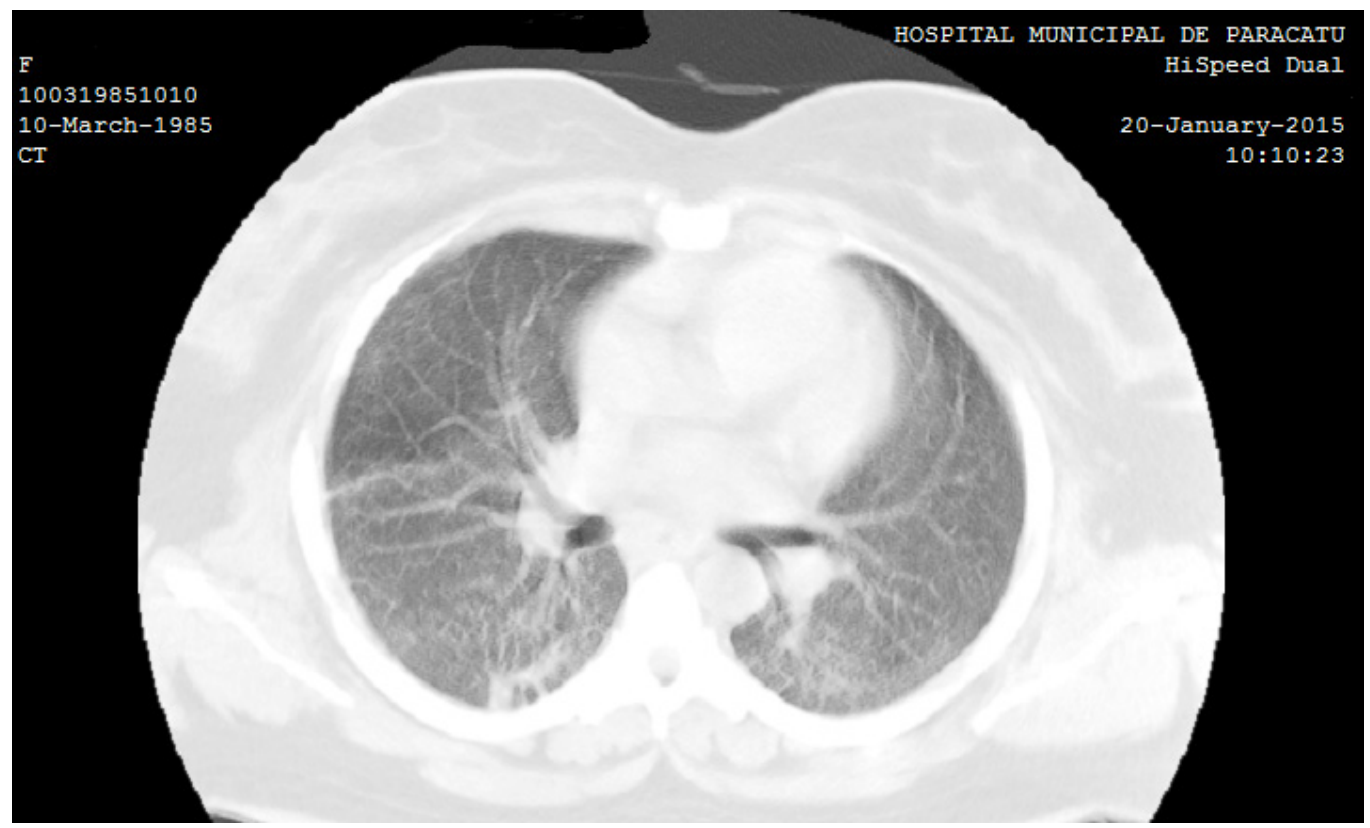

Opacidade em vidro fosco em ambos os pulmões.

Figura 1: Tomografia Computadorizada de Tórax

Iniciou o tratamento com Sulfametoxazol+ Trimetoprima 80mg-400mg três ampolas EV de 06/06 horas por 21 dias; Nistatina suspensão oral 08/08 horas $100.000 \mathrm{UI} / \mathrm{ml}$ por 21 dias; Hidrocortisona 100mg EV de $8 / 8 \mathrm{~h}$ horas por 21 dias. Iniciou a terapia antirretroviral (TARV) com uso de Lopinavir+Ritonavir (LPV/r) 80mg $+20 \mathrm{mg}$, Zidovudina (AZT) 300mg, Lamivudina (3TC) $150 \mathrm{mg}$.

Paciente em uso de máscara de $\mathrm{O}_{2}$ à $7 \mathrm{~L} / \mathrm{min}$, saturando 95\%, apresentou melhora da dispneia; no segundo dia de internação, foi substituído máscara de $\mathrm{O}_{2}$ por cateter nasal à $3 \mathrm{~L} / \mathrm{min}$, apresentou boa saturação $96 \%$ e melhora do desconforto respiratório.
Evoluiu com melhora clínica da dispneia e da monilíase oral, recebendo alta após terapia antimicrobiana, sendo orientada a continuar o acompanhamento pré-natal, além do uso contínuo da TARV.

Foi mantido antibiótico, corticóide sistêmico, Nistatina por 21 dias e a TARV durante toda a gestação. Além de iniciar a rotina do pré-natal até o dia do parto. Após o parto, investigou-se a infecção pelo vírus HIV na criança, os exames não apresentaram alterações.

\section{DISCUSSÃO}

Gestantes como a do caso descrito, que não realizam 
a rotina Pré-Natal estão susceptíveis a infecções, estas representam um grave problema no período gestacional, podendo alterar o curso clínico da gestação, levando a um pior prognóstico materno-fetal.

A infecção causada pelo HIV pode gerar alterações na mãe e no feto; é de suma importância a realização do Pré-Natal, pois este propicia um diagnóstico precoce, podendo alterar o curso da doença na mãe, evitando assim a transmissão vertical para o feto.

O Projeto Nascer do Ministério da Saúde recomenda a triagem sorológica anti-HIV para toda gestante durante o pré-natal, possibilitando o diagnóstico da infecção e a profilaxia da transmissão vertical. Desse modo a maioria das mulheres infectadas é identificada durante a gestação ou no momento do parto 9 .

A paciente não realizou as consultas do pré-natal e a sorologia para HIV, porém foi hospitalizada, pois apresentava doença definidora de SIDA: pneumocistose. O diagnóstico de HIV/SIDA e de pneumocistose ocorreu após internação, o que evidencia um pior prognóstico maternofetal, pois caso o pré-natal fosse realizado, a infecção pelo vírus HIV teria sido diagnosticada precocemente, e a terapia antirretroviral (TARV) instalada, de modo que no momento do parto, o risco de transmissão vertical diminuiria substancialmente.

Aproximadamente 38 a $48 \%$ das gestantes ainda chegam às maternidades sem o resultado da sorologia anti-HIV do pré-natal, sendo necessária a realização teste rápido no momento do parto, isto faz com que as ações de prevenção da transmissão vertical levem mais tempo para serem realizadas ${ }^{10,11}$.

O manual "Recomendações para Profilaxia da Transmissão Vertical e Terapia Antirretroviral em Gestantes do Ministério da Saúde preconiza que, se possível, a sorologia deva ser repetida no terceiro trimestre de gestação, desse modo o tempo para iniciar as medidas de transmissão vertical do HIV diminui substancialmente ${ }^{12}$.

O P. jirovecii é um patógeno oportunista, eucariota unicelular, de difícil cultivo e com especificidade restrita aos seres humanos. Apresenta tropismo pelo pulmão, gera uma pneumonia intersticial, conhecida como pneumocistose, também chamada de pneumonia por Pneumocystis (PCP) em imunocomprometidos, a infecção é assintomática em imunocompetentes ${ }^{13,14}$.

A pneumocistose é a causa mais comum de doença pulmonar oportunista em pacientes com SIDA, cuja contagem de CD4+ está abaixo de $200^{16}$. É uma doença grave, com alta mortalidade, que cursa com hipoxemia grave, de modo que na maioria das vezes o paciente necessita de ventilação mecânica. Sendo assim é necessário realizar o tratamento da SIDA e da doença pulmonar oportunista $^{15,16}$.

O achado mais característico de pneumocistose evidente na tomografia computadorizada de tórax é a presença de áreas com atenuação em vidro fosco, ou seja, há o aumento homogêneo da atenuação sem obscurecer as imagens vasculares ${ }^{17}$. $\mathrm{O}$ achado radiográfico quando associado à toxemia, evidente na gasometria arterial, e o aumento de DHL sérica em paciente com SIDA é muito sugestivo de pneumocistose ${ }^{3}$.

A broncoscopia com lavado broncoalveolar (LAB) é o padrão ouro para o diagnóstico de pneumocistose apresentando uma sensibilidade de $90-98 \%$, todavia não é realizado com frequência, devido o alto custo e a necessidade de equipe especializada e materiais especiais.

O exame histopatológico evidencia exsudato intraalveolar espumoso, um líquido proteináceo que contém parasitos, fibrina e restos celulares; sendo que o acúmulo deste exsudato gera o padrão em vidro fosco visualizado na TC de tórax ${ }^{3,17}$.

As manifestações clínicas apresentam inicio insidioso, com duração dos sintomas por um período de semanas a meses até a hospitalização. O paciente apresenta dispneia progressiva, febre, tosse seca, desconforto torácico. Ao exame físico, os achados de taquipneia, taquicardia, ausculta pulmonar sem alterações são comuns, porém crepitação à ausculta pulmonar ocorre em 30\% a $40 \%$ dos casos, este achado indica maior gravidade; além disso, a monilíase oral é a co-infecção mais comum, sendo considerada um achado quase universal ${ }^{16}$.

O tratamento é guiado pela gravidade do quadro clínico apresentado pelo paciente. A pneumocistose é classificada em leve a moderada $(\mathrm{PaO} 2 \geq 70 \mathrm{mmHg})$, sendo o tratamento instituído por via oral; e moderada a grave $(\mathrm{PaO} 2<70 \mathrm{mmHg})$, tratamento por via endovenosa. A primeira escolha é Sulfametoxazol + Trimetroprima, com 15-20mg de Trimetoprima $/ \mathrm{kg} / \mathrm{dia}$ oral a cada seis ou oito horas por 21 dias $^{18}$.

Os corticosteróides podem ser associados ao antibiótico, quando $\mathrm{PaO} 2<70 \mathrm{mmHg}$ em gradiente de ar ou alveolares-capilar $>35 \mathrm{mmHg}$, a primeira escolha é Prednisona, podendo ser usado Metilprednisolona como substituto. Deve se ressaltar que o início do tratamento com corticosteróides deve ser realizado em associação com o antibiótico ${ }^{18}$.

A pneumocistose apresenta um curso clinico mais agressivo durante o período gestacional, com aumento da morbimortalidade. Desfechos maternos e fetais permanecem sombrios em relação à infecção no período gestacional. O tratamento com SulfametoxazolTrimetoprim, quando comparado com outras terapias, pode resultar em um melhor resultado terapêutico ${ }^{19}$.

Mais de $80 \%$ de mulheres com AIDS estão em idade reprodutiva. A pneumocistose é a causa mais comum de morte relacionada à AIDS em mulheres grávidas nos Estados Unidos ${ }^{19}$. Faltam dados na literatura brasileira sobre pneumocistose em gestantes soropositivas.

A taxa de mortalidade é mais elevada em mulheres grávidas com HIV e pneumocistose, do que a observada em indivíduos infectados pelo HIV com pneumocistose. 
Diferentes estudos relataram taxas de mortalidade variando de 1 a $16 \%{ }^{19,20}$.

A pneumonia fúngica causada pelo $P$. jirovecii no primeiro e segundo trimestres gestacionais confere pior prognóstico materno e fetal. Os desfechos maternos e fetais foram melhores nos casos de pneumocistose durante o terceiro trimestre da gestação. A apresentação clínica não é alterada pela gestação, sendo semelhante a outros casos de pneumocistose em pacientes infectados pelo $\mathrm{HIV}^{19,22}$. $\mathrm{O}$ tratamento com Sulfametoxazol-Trimetoprim associa-se ao aumento da taxa de sobrevivência materno-fetal, como observado no caso descrito.

A realização do teste de HIV na rotina Pré-Natal é essencial para identificar as mulheres infectadas pelo vírus, dessa maneira, identifica pacientes que devem iniciar a TARV, visando diminuir o risco de transmissão vertical do vírus HIV, além de melhorar a saúde materna ${ }^{23}$.

A infecção pelo HIV quando bem controlada melhora a qualidade de vida do indivíduo. A não adesão ao tratamento provoca resistência do vírus HIV, às drogas hoje utilizadas, além de predispor à infecções oportunistas. Sendo assim, o diagnóstico e tratamento precoce aumentam a sobrevida do paciente e impede que se instalem doenças oportunistas. É de suma importância a realização das consultas de pré-natal, pois garante uma gestação segura para mãe e filho.

Conflitos de interesse: Os autores declaram não haver conflitos de interesse.

Agradecimentos: Agradecemos à Dr ${ }^{\mathrm{a}}$ Fernanda Beatriz Ferreira Abdulmassih, médica ginecologista e Professora Titular do Departamento de Ginecologia e Obstetrícia da Faculdade Atenas e à $\mathrm{Dr}^{\mathrm{a}}$ Rosekeila Simões Nomelini, médica ginecologista e Professora Adjunta da Universidade Federal do Triângulo Mineiro, UFTM, pela contribuição na busca de artigos para elaboração do manuscrito.

\section{REFERÊNCIAS}

1. D'Angelo E, Calderini E, Robatto FM, Puccio P, Milic-Emili $\mathrm{J}$. Lung and chest wall mechanics in patients with acquired immunodeficiency syndrome and severe Pneumocystis carinii pneumonia. Eur Respir J. 1997;10:2343-50. doi: 10.1183/09031936.97.10102343.

2. Thomas CF, Limper AH. Pneumocystis pneumonia. N Engl J Med. 2004;350:2487-98. Available from: http://paetc. org/wp-content/uploads/2013/04/Thomas_PCP-review NEJM_2004.pdf.

3. Miller R. HIV-associated respiratory diseases. Lancet. 1996;348:307-12. http://dx.doi.org/10.1016/S01406736(95)11037-2.

4. Feikin DR, Feldman C, Schuchat A, Janoff EN. Global strategies to prevent bacterial pneumonia in adults with HIV disease. Lancet Infect Dis. 2004;4:445-55. http://dx.doi. org/10.1016/S1473-3099(04)01060-6.

5. Gordin FM, Roediger MP, Girard PM, Lundgren JD, Miro JM, Palfreeman A, Rodriguez-Barradas MC, Wolff MJ, Easterbrook PJ, Clezy K, Slater LN. Pneumonia in HIVinfected persons: increased risk with cigarette smoking and treatment interruption. Am J Respir Crit Care Med. 2008;178:630-6. doi: 10.1164/rccm.200804-617OC.

6. Benito-Hernández N, Moreno-Camacho A, Gatell-Artigas JM. Complicaciones infecciosas pulmonares en los pacientes con infección por el virus de la inmunodeficiencia humana en la era del tratamiento antirretroviral de gran actividad en España. Med Clin (Barc). 2005;125(14):548-55. doi: $10.1157 / 13080461$.

7. Benito N, Moreno A, Miro JM, Torres A. Pulmonary infections in HIV-infected patients: an update in the 21 st century. Eur Respir J. 2012;39(3):730-45. doi: 10.1183/09031936.00200210.

8. Swaminathan S, Padmapriyadarsini C, Narendran G. HIVassociated tuberculosis: clinical update. Clin Infect Dis. 2010;50(10):1377-86. doi: 10.1086/652147.
9. Sonnenberg P, Glynn JR, Fielding K, Murray J, GodfreyFaussett P, Shearer S. How soon after infection with HIV does the risk of tuberculosis start to increase? A retrospective cohort study in South African gold miners. J Infect Dis. 2005;191(2):150-8. doi: 10.1086/426827.

10. Marchiori E, Pereira CIGS, Moreira LBM, Capone D, Moraes HP de. Pneumocistose na síndrome da imunodeficiência adquirida: correlação da tomografia computadorizada de alta resolução com a anatomopatologia. Radiol Bras. 2001;34(6):317-21. http://dx.doi.org/10.1590/S010039842001000600003 .

11. Wakefield AE. Pneumocystis carinii. Br Med Bull. 2002;61(1):175-88. doi: 10.1093/bmb/61.1.175.

12. Furrer H, Opravil M, Bernasconi E, Telenti A, Egger M. Stopping primary prophylaxis in HIV-1-infected patients at high risk of toxoplasma encephalitis. Swiss HIV Cohort Study. Lancet. 2000;355(9222):2217-8.

13. Opportunistic Infections Project Team of the Collaboration of Observational HIV Epidemiological Research in Europe (COHERE), Mocroft A, Reiss P, Kirk O, Mussini C, Girardi E, Morlat P, Stephan C, De Wit S, Doerholt K, Ghosn J, Bucher HC, Lundgren JD, Chene G, Miro JM, Furrer H. Is it safe to discontinue primary Pneumocystis jiroveci pneumonia prophylaxis in patients with virologically suppressed HIV infection and a CD4 cell count $<200$ cells/microL. Clin. Inf. Dis. 2010;51(5):611-9. doi: 10.1086/655761.

14. Morris A, Lundgren JD, Masur H, Walzer PD, Hanson DL, Frederick T, Huang L, Beard CB, Kaplan JE. Current epidemiology of Pneumocystis pneumonia. Emerg Infect Dis. 2004;10(10):1713-20. doi: 10.3201/eid1010.030985.

15. Kuhlman JE. Pneumocystic infections: the radiologist's perspective. Radiology. 1996;198(3):623-35. doi: 10.1148/ radiology.198.3.8628844.

16. Brasil. Ministério da Saúde. Projeto Nascer. Portaria 2104 de 19 de novembro de 2002: institui, no âmbito do Sistema 
Único de Saúde - SUS -, o Projeto Nascer-Maternidades e dá outras providências. Diário Oficial da União - DOU, 2002 nov 21; Secção 1: 25.

17. Morimura MCR, Mendes MDC, Souza AI, Alencar LCA. Frequência de testagem rápida para o HIV durante a admissão para o parto em puérperas no Instituto Materno Infantil Prof. Fernando Figueira, IMIP. Rev Bras Saúde Matern Infantil. 2006;6(Supl 1):S69-S76. http://dx.doi.org/10.1590/S151938292006000500010 .

18. Brasil. Ministério da Saúde. Monitoraids. Brasília (DF); dezembro de 2004

19. Brasil. Ministério da Saúde. Coordenação Nacional de DST/ AIDS. Recomendações para profilaxia da transmissão vertical e terapia anti-retroviral em gestantes. Brasília (DF); 2006. Disponível em: http://www.aids.gov.br/sites/default/files/ consenso_gestantes_2010_vf.pdf.

20. Matos O, Costa MC, Correia I, Monteiro P, Vieira JR, Soares J, Bonnet M, Esteves F, Antunes F. Pneumocystis jiroveci infection in immunocompetent patients with pulmonary disorders, in Portugal. Acta Med Port. 2006;19:121-6. Disponível em: http://www.actamedicaportuguesa.com/ revista/index.php/amp/article/view/919/592.

21. Gajdusek DC. Pneumocystis carinii as the cause of human disease: historical perspective and magnitude of the problem: introductory remarks. Natl Cancer Inst Monogr. 1976;43:1-11.

22. Thomas CF Jr, Limper AH. Pneumocystis pneumonia. N Engl J Med. 2004;350(24):2487-98. doi: 10.1056/NEJMra032588.

23. Calderon EJ, Gutiérrez-RiveroS, Durand-Joly I, Dei-Cas E. Pneumocystis infection in humans: diagnosis and treatment. Expert Rev Anti Infect Ther. 2010;8(6):683-701. doi: 10.1586/ eri.10.42.
24. Pereira CIGS, Marchiori E, Souza Jr AS. Pneumocistose no paciente com síndrome da imunodeficiência adquirida: avaliação pela tomografia computadorizada de alta resolução do tórax. Rev Imagem. 2001;23:91-9.

25. Centers for Disease Control and Prevention. Guidelines for Prevention and Treatment og Opportunistic Infections in HIV Infected Adults and Adolescents. Recommendations from CDC, the National Institutes of Health, and the HIV Medicine Association of the Infections Diseases Society of America. MMWR. 2009;58:6-10.

26. Ahmad H, Mehta NJ, Manikal VM, Lamoste TJ, Chapnick EK, Lutwick LI, Sepkowitz DV. Pneumocystis carinii pneumonia in pregnancy. Chest. 2001;120(2):666-71. http:// dx.doi.org/10.1378/chest.120.2.666.

27. Bozzette SA, Finkelstein DM, Spector SA, et al. A randomized trial of three antipneumocystis agents in patients with advance human immunodeficiency virus infection. N Engl J Med. 1995;332:693-9. doi: 10.1056/NEJM199503163321101.

28. Bozzette SA, Sattler FR, Chiu J, et al. A controlled trial of adjunctive treatment with corticosteroids for Pneumocystis carinii pneumonia in the acquired immunodeficiency syndrome. N Engl J Med. 1990;323:1451-7. doi: 10.1056/ NEJM199011223232104.

29. Kovacs JA, Hiemenz JW, Macher AM, et al. Pneumocystis carinii pneumonia: a comparison between patients with the acquired immunodeficiency syndrome and patients with other immunodeficiencies. Ann Intern Med. 1984;100:663-71.

30. Drake AL, Wagner A, Richardson B, John-Stewart G. Incident HIV during pregnancy and postpartum and risk of mother-to-child HIV transmission: a systematic review and meta-analysis. PLoS Med. 2014;11(2):e1001608. doi: 10.1371/journal.pmed.1001608. 